\title{
HARDWARE IMPLEMENTATION OF CONCURRENT PERIODIC EFSMS
}

\author{
Hisaaki Katagiri ${ }^{1}$, Masayuki Kirimura ${ }^{1}$, \\ Keiichi Yasumoto ${ }^{2}$, Teruo Higashino ${ }^{1}$ and Kenichi Taniguchi ${ }^{1}$ \\ ${ }^{1}$ Graduate School of Eng. Sci., Osaka Univ., Toyonaka, Osaka 560-8531, JAPAN \\ 2 Dept. Info. Proc. \& Man., Shiga Univ., Hikone, Shiga 522-8522, JAPAN \\ yasumoto@biwako.shiga-u.ac.jp, (kirimura,higashino,taniguchi)@ics.es.osaka-u.ac.jp
}

\begin{abstract}
This paper proposes a concurrent periodic EFSMs model and a technique to synthesize hardware circuits from real-time system specifications in this model. In the proposed model, the data exchange by synchronous execution of the same events in multiple EFSMs (multiway synchronization) can be specified like LOTOS. The executable time range of each event can be specified as a logical conjunction of linear inequalities of the execution time of its preceding events, constants and integer variables with some values input from environments. Here, we assume that every event sequence starting from the initial state in each EFSM returns to the initial state in the specified time interval. The proposed synthesis technique implements only executable combination of branches in a given specification with a schedule table which guarantees that if each $\mathrm{I} / \mathrm{O}$ event is executed within the specified time range, at least one of the following event sequences remains executable. Some experiments show that the performance and size of the generated circuits are reasonable for practical use.
\end{abstract}

Keywords: Real-time systems, hardware implementation, scheduling, multi-way synchronization, E-LOTOS

\section{INTRODUCTION}

For the needs of high-speed computer networks, hardware implementation techniques for communication systems have become important. In general, in development of high performance communication systems, we repeatedly modify parameters such as timing restriction for each task or I/O action as well as internal algorithms and structures of the systems, and simulations are carried out until the expected performance is achieved. Therefore, in order to reduce development costs, rapid pro-

The original version of this chapter was revised: The copyright line was incorrect. This has been corrected. The Erratum to this chapter is available at DOI: 10.1007/978-0-387-35533-7_26

Tommaso Bolognesi and Diego Latella (Eds.), Formal Methods for Distributed System Development. (c) 2000 IFIP International Federation for Information Processing.

Published by Kluwer Academic Publishers. All rights reserved. 
totyping techniques for automatic synthesis of hardware circuits from high-level specifications are essential in early stages of development [11].

In recent years, high-level synthesis techniques using FDT have been stressed. $[1,13]$ have proposed hardware implementation techniques using SDL and Estelle, respectively, and [8, 10,14] have proposed techniques based on LOTOS [5]. However, those existing techniques do not handle timing constraints for event execution nor guarantee real-time processing of tasks in the generated circuits. [2] has proposed a technique for guaranteeing such real-time processing. This technique supposes that multiple concurrent processes are given as the corresponding task graphs and calculates an optimal scheduling where the total cost of processors is minimized under the condition that the deadlines of all tasks are met. Unfortunately, it may be difficult to apply this technique to development of real-time communication systems because it does neither handle communication among concurrent processes nor consider execution timings of $\mathrm{I} / \mathrm{O}$ events.

This paper proposes a model called concurrent periodic EFSMs and a technique to synthesize hardware circuits from real-time system specifications described in this model. In the proposed model, like LOTOS the data exchange by synchronous execution of the same events in multiple EFSMs (called multi-way synchronization) can be specified, and like ELOTOS [6] a timing constraint represented as a logical conjunction of linear inequality expressions of the execution time of preceding events, constants and integer variables (where some values are input from external environments) can be specified to decide an executable time range of each event. In the proposed model, we assume that each branch of event sequences starting from the initial state must return to the initial state after executing some events in the specified time interval. Since most of real-time and multimedia systems consist of periodic behavior, this assumption seems reasonable for development of such systems.

The following criteria should be considered when we synthesize circuits from specifications in the proposed model: (1) guarantees for deadlock-free execution of event sequences, (2) fairness in selection of all executable branches, (3) assignment of a possibly wide time range to each I/O event execution. Therefore, the proposed synthesis technique generates circuits where all executable branches in the given specification can be selected, and only schedulable event sequences are dynamically executed depending on the execution time of the preceding events. For such guarantees, the proposed technique derives an appropriate executable time range of each event with a technique for solving integer linear programming problems (ILP). Here, we maximize the total sum of executable time ranges of $\mathrm{I} / \mathrm{O}$ events. 


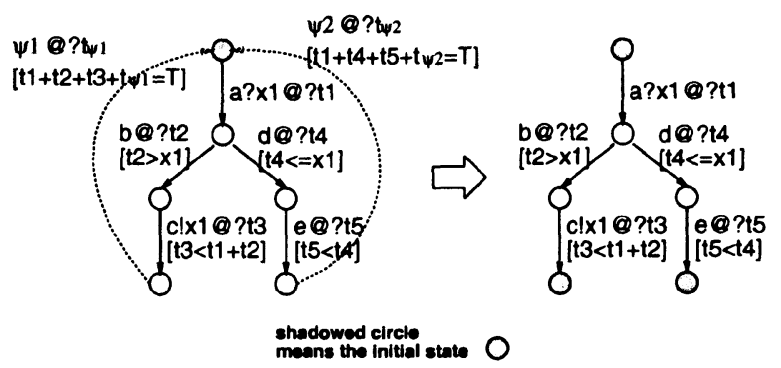

Figure 1 Periodic EFSM

Based on the techniques we previously proposed in [7, 14], a given specification in the proposed model is implemented as a hardware circuit where it consists of sequential circuits corresponding to EFSMs and a combinational logic circuit for the controller of multi-way synchronization among EFSMs. For timing guarantees, we construct a scheduling circuit indicating schedulable event sequences at each time.

To evaluate the proposed technique, we have implemented tools to generate the corresponding RTL-level VHDL descriptions. Through some experiments, we have confirmed that the performance and size of the generated circuits are reasonable for practical use.

\section{CONCURRENT PERIODIC EFSMS}

EFSM is defined as 5-tuple $<S, I, V$, clock, $\delta$, init $>$. Here, $S$ is a finite set of states, $I$ is a finite set of events (i.e., transitions), $V$ is a finite set of variables, clock is a counter holding the waiting time since the EFSM has moved to a new state, $\delta$ is a finite set of transition rules, and init represents the initial state and initial values of all variables.

It is assumed that the execution of each event is completed in a moment. The event which exchanges data between the system and its external environment is called an I/O event, and the event which is executed in synchronization with the same one of other EFSMs is called a synchronous event. Like the notation of LOTOS, the input and output events are represented as $a ? x$ and $b ! E$, respectively. Here, $a$ and $b$ denote gate names for input and output, respectively. In addition, in one event, multiple inputs/outputs such as " $a$ ? $x ! 10$ " can be specified. Moreover, like E-LOTOS, $a @$ ? $t$ denotes that the value of $c l o c k$ at the execution time of event $a$ (called delay time) is substituted to time variable $t$. The value of clock is treated as an integer. 
Each transition rule is defined as 4-tuple $<s_{c u r}, s_{\text {next }}$, a, guard $>$ $\left(s_{\text {cur }}, s_{\text {next }} \in S, a \in I\right)$. Here, guard is called a transition condition, and it is specified by a logical expression consisting of the input/time variables used in the EFSM. In each EFSM, arbitrary types of variables such as integer, bool and string can be specified. However, the types of all variables used in the transition conditions must be integer. If the value of the transition condition guard is true at state $s_{c u r}$ and event $a$ is executed, then the EFSM moves to state $s_{n e x t}$. By using time variables in transition conditions, we can give a restriction for the executable time of each event. For example, $a ? x @ ? t[(2 \leq t) \operatorname{and}(0 \leq x) a n d(x \leq 10)]$ denotes that input event $a ? x$ must be executed after waiting at least 2 units of time in the current state, and that an integer satisfying the condition $(0 \leq x)$ and $(x \leq 10)$ is input from gate $a$ and it is substituted into input variable $x$. As a transition condition, only a logical conjunction of integer linear inequality expressions consisting of time variables representing the delay time of the preceding events and/or input variables can be specified. If multiple events are executable at the current state, only one event is selected and executed non-deterministically.

If a given EFSM satisfies the following restrictions, then the EFSM is called a periodic EFSM.

- All paths (event sequences) from the initial state return to the initial state.

- The transition conditions are specified so that even if any path from the initial state is selected, it can return to the initial state in a certain fixed time interval $T$.

The graph of the left-hand side in Fig. 1 shows an example periodic EFSM. In this EFSM, at first, a value is input from gate $a$ and substituted to variable $x 1$ at time $t 1$. Then, until $x 1$ units of time has passed, event $d$ can be executed. After $x 1$ units of time has passed, event $b$ can be executed. If event $b$ is executed, then the delay time of event $b$ is substituted into time variable $t 2$, and event $c ! x 1 @ ? t 3$ becomes executable. In order to make the EFSM return to the initial states in the specified time interval $T$, we assume that every path (event sequence) from the initial state has the special dummy transition $\psi$ as the last event of the path where the transition condition of the dummy transition $\psi$ gives the timing constraint to make the path return to the initial state in the specified time interval $T$. In Fig. 1, such dummy transitions $\psi_{1}$ and $\psi_{2}$ are shown as dotted edges. For the sake of simplicity, hereafter, we will omit such dummy transitions in the graphs and depict the graphs in the form of the right-hand side in Fig. 1.

The synchronization relation among EFSMs is specified as follows using the parallel operators in LOTOS. 


\section{$S::=S \mid[$ gate_list $]|S \quad| \quad S \||| S|(S) \quad| \quad E F S M$}

Here, EFSM is a name of EFSM, and gate_list is a gate list of the events to be synchronized between its operator's both sides of EFSMs.

For example, in $M 1|[a, b]|(M 2|[b]| M 3)$ of Fig. 4, event $a$ must be executed simultaneously either between $M 1$ and $M 2$ or between $M 1$ and $M 3$. When synchronous events $a$ of $M 1$ and $M 2$ are executed simultaneously, the output value $x 1-2$ of event " $a ! x 1-2 @$ ? $t 3$ " of $M 1$ is substituted to variable $x 3$ of event " $a$ ? $x 3 @$ ? $t 10$ " of $M 2$.

The details about the transition conditions for synchronous events are omitted here, since they are the same as those of LOTOS [5].

\section{BASIC POLICY OF CIRCUIT DERIVATION}

\section{Static Scheduling Policy}

In order to derive a hardware circuit from a given specification, it is important to decide when we should execute each event so that it can satisfy the timing constraints described in the specification.

For example, consider the following event sequence.

$$
s 1 \stackrel{a @ ? t_{a}\left[t_{a} \geq 2\right]}{\longrightarrow} s 2 \stackrel{b \Theta ? t_{b}\left[t_{a}+t_{b} \leq 5\right]}{\longrightarrow} s 3
$$

Since the timing constraint $t_{a} \geq 2$ of event $a$ means that event $a$ can always be executed after the delay time at state $s 1$ becomes 2 , event $a$ can be executed, for example, at delay time 10. However, it does not satisfy the constraint $t_{a}+t_{b} \leq 5$ of the following event $b$ in this case.

On the other hand, when event $a$ is executed at delay time 2, event $b$ can be executed at any delay time between 0 and 3 . If all events in a given event sequence can be executed at the time satisfying given timing constraints, then we will say that the event sequence is schedulable. In order to make each event sequence schedulable, we must decide the execution time of each event so that the succeeding events can satisfy their timing constraints.

Then, the proposed technique derives the range of the execution time of each event which makes the whole event sequence schedulable. Note that generally such a range may become narrow compared with that of the given specification. Moreover, there exists a case where the range of the execution time of the succeeding event is dynamically changed depending on the execution time of its preceding events. In the above example, if event $a$ is executed at delay time $h(2 \leq h \leq 5)$, then event $b$ is executable at any delay time between 0 to $(5-h)$. Since such a dynamic scheduling is not easy to implement as a hardware circuit, we will adopt a 
static scheduling policy in this paper. Therefore, the proposed technique derives the constant time range for each event where the derived time range guarantees that if each event is executed at any time in the range, the succeeding events can also be executed at any time in the derived time ranges of those events. For example, for the above example, if we give a schedule so that $2 \leq t_{a} \leq 3$ and $0 \leq t_{b} \leq 2$ hold, this event sequence is always executable. Using such a static scheduling policy, it becomes possible to judge the executability of each event by using only one global timer representing the current time and some simple logic circuits. Also, we do not need to keep the actual execution time of all events unlike a dynamic scheduling policy.

\section{Requirements for Derived Circuits}

Our synthesis technique derives circuits which satisfy the following requirements.

- In order to make given concurrent EFSMs periodic, only schedulable sets of paths (event sequences) in a given specification must be selected and executed.

- It is desirable to derive a possibly wide range of the execution time of each event.

Since each EFSM has some branches as its event sequences where some of them may include synchronous events, the following problems should be considered to satisfy the above requirements.

Like the example in Fig. 2, if there are conditional branches after an $\mathrm{I} / \mathrm{O}$ event $a$ is executed, the range of executable time of event $a$ may differ depending on which conditional branch is selected. Note that, as pointed out in Fig. 2, as time passes, some of conditional branches may become un-executable (un-schedulable). Moreover, like the example in Fig. 3, synchronous event $b$ of EFSM1 becomes executable after $a$ is executed, and it can synchronize with either of two synchronous events $b$ in EFSM2. Depending on which branch $\alpha$ or $\beta$ is selected, the range of executable time of the preceding event $a$ may differ. Then, in the proposed technique, we find all possible sets of paths (event sequences) of concurrent EFSMs, and derive the range of the execution time of each event depending on which set of paths is selected. In the derived circuits, at each unit of time, the controller checks which path becomes un-schedulable and it makes only schedulable paths executable.

\section{DERIVATION OF EXECUTABLE TIME RANGE OF EACH EVENT}

In this section, in order to derive the executable time range of each event, we construct linear inequality expressions from the information about tuples of synchronous events (which are executed simultaneously 

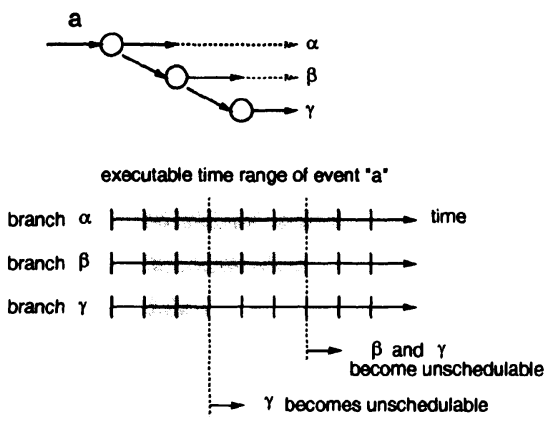

Figure 2 Executable time range of an event followed by branches

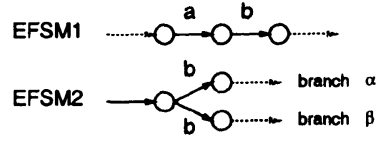

EFSM1 [b]| EFSM2

executable time range of event " $a$ " of EFSM1

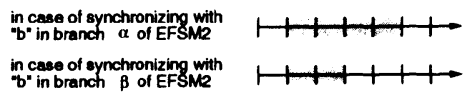

Figure 3 Executable time range of an event depending on selection of multi-way synchronization

among EFSMs), temporal ordering of events in each EFSM, and the timing constraint of each event. Since some input variables may influence the execution of event sequences (e.g., the cases that the variables are used in guard expressions or in timing constraints of events), we also derive allowable value ranges for those variables.

\subsection{DERIVATION OF TUPLES OF SYNCHRONOUS EVENTS}

By tracing executable events in the tuple of all EFSMs sequentially from the initial global state, we can derive the information about in what combination of EFSMs and in what tuple, events must be executed in synchronization with each other. Several techniques for such reachability analysis have been proposed for models including event synchronization among concurrent processes, for example, in [12]. We can use the techniques to derive such information about synchronization of events. However, since reachability analysis generally needs to explore $n^{m}$ global states in the worst case (here, $n$ and $m$ represent the number of states in each EFSM and the number of synchronizing EFSMs, respectively), computation time grows exponentially as the number of parallel processes increases. Also, in EFSMs, it may take much time to check execution conditions (i.e., boolean expressions) for synchronizations.

In our concurrent periodic EFSMs model, since each EFSM executes one of its event sequences in a specified time interval and all EFSMs have the same time interval, due to restrictions explained in Sect. 2, we can easily derive synchronous event tuples by simply checking whether the synchronous events appear in the same order for each combination of event sequences (due to space limitation, the detailed procedure is 
omitted). So, in our model, computation time for deriving synchronous event tuples is bound to $N \cdot B^{m}$ where $N$ is the maximum number of events in each event sequence, and $B$ is the maximum number of branches in each EFSM. In general, $N \cdot B^{m} \ll n^{m}$ holds.

\subsection{CONSTRUCTION OF ILP PROBLEMS}

We use the execution time of each event as a parameter of the ILP problem. We also use the allowable value for each input variable as a parameter. From the set of event sequences $E S_{i}$ of each $E F S M_{i}$ and the set of tuples of synchronous events denoted by $S Y N$, we construct the following logical conjunction of linear inequalities as the ILP problem. Hereafter, we denote the execution time of event $a$ by $T_{a}$, and the allowable value of variable $x$ by $V_{x}$. We assume that each time variable is counted as the time elapsed from the beginning of the current time interval. We use concurrent periodic EFSMs in Fig. 4 as an example.

(1) constraints for temporal ordering of events in each EFSM

Execution time of an event must be less than execution time of the succeeding events. Therefore, for example, for event sequence $P 12$ of $M 1$ in Fig. 4, the following constraint is derived.

$$
T_{c}<T_{e}<T_{b}
$$

(2) constraints for tuples of synchronous events

All events of each tuple of synchronous events in $S Y N$ must be executed at the same time. So, for example, the following constraint is derived for event $b$ executed synchronously among event sequences $\{\mathrm{P} 12$, P21, P33\} in Fig. 4.

$$
T_{b_{M 1}}=T_{b_{M 2}}=T_{b_{M 3}}
$$

(3) timing constraints attached to each event

Timing constraints attached to each event are included as it is in the ILP program. However, since each original time variable represents the delay time in the current state, it must be replaced by the corresponding variable representing the time elapsed from the beginning of the current time interval.

For example, the timing constraint of event $b$ in the following event sequence (corresponding to $P 12$ in Fig. 4)

$$
c ? x 1 @ ? t 1[\ldots] ; e @ ? t 4[\ldots] ; b @ ? t 5[t 5<t 1+t 4]
$$

is represented as

$$
\begin{array}{r}
\left(T_{b}-T_{e}\right)<T_{c}+\left(T_{e}-T_{c}\right) \\
\Rightarrow T_{b}-2 T_{e}<0
\end{array}
$$


by replacing $t 1, t 4$ and $t 5$ by $T_{c}, T_{e}-T_{c}$ and $T_{b}-T_{e}$, respectively.

(4) constraints for the allowable value of each integer variable

Each input variable used in some timing constraints of events should have a certain value which satisfies those constraints.

In $P 11$ of Fig. 4 , event $c ? x 1 @ \ldots$ inputs some value to the variable $x 1$, and $x 1$ is used in the timing constraint of the next event $d @ ? t 2[t 2>x 1]$. Thus, in this case, the following constraint for $x$ is derived.

$$
T_{d}-T_{c}>V_{x 1}
$$

In addition, event $a !(x 1-2)$ of $P 11$ is specified to synchronize with either $a ? x 3 \ldots[(x 3>x 2)$ and...] of $P 22$ or $a ? x 4 \ldots[t 15<x 4+5]$ of $P 32$. So, the following constraint for the variable $x 1$ is derived for the case that synchronization of $a$ is executed between $P 11$ and P22.

$$
V_{x 1}-2=V_{x 3}
$$

As we explained in Sect. 3, the executable time range of each event may differ depending on what branch is selected and executed. So, in the proposed technique, we construct the above constraint expression for each branch (i.e., each event sequence from the initial state), and solve the corresponding ILP problem to obtain the executable time of each event in the sequence. Similarly, when an event sequence includes a synchronous event, its executable time range may also differ depending on what combination of synchronous events is executed. For example, event $a !(x 1-2)$ in sequence $P 11$ in Fig. 4 can be executed in synchronization with either $a ? x 3 \ldots$ in $P 22$ or $a ? x 4 \ldots$ in $P 32$, where the executable time range of $a$ in $P 11$ differs depending on which $a$ is synchronized. Thus, we derive each case of the executable time range by constructing the constraint expression for each combination.

\subsection{DERIVATION OF AN EXECUTABLE TIME RANGE OF EACH EVENT}

In the constraint expression constructed in Sect. 4.2, we used variables representing the "execution time" of events. To obtain the "executable time range", we modify the expression as follows.

As we explained in Sect. 3, we derive the upper and lower bounds of executable time of each $\mathrm{I} / \mathrm{O}$ event as constants. For the purpose, in the constraint expression defined in Sect. 4.2, we replace variable $T_{a}$ (representing the execution time of event $a$ ) by $T_{a_{\min }}$ and $T_{a_{\max }}$ which represent the lower and upper bounds of execution time, respectively. Also, for each variable which influences the executable time ranges of some events or the selection of event sequences, we similarly derive the 
lower and upper bounds for its allowable value range. Hereafter, we denote the execution time of $a_{k}$ by $T_{k}$ in event sequence $a_{1} ; a_{2} ; \ldots ; a_{n}$, and the lower and upper bounds of $T_{k}$ by $T_{k_{m i n}}$ and $T_{k_{\max }}$, respectively (for each variable, we use $V_{k_{\min }}$ and $V_{k_{\max }}$ ).

First, we add the following constraint so that the executable time ranges of a series of events in each event sequence does not have common ranges.

$$
T_{1_{\min }} \leq T_{1_{\max }}<T_{2_{\min }} \leq T_{2_{\max }}<\cdots<T_{n_{\min }} \leq T_{n_{\max }}
$$

Next, the constraint for synchronous event $a_{j}$ which synchronizes with event $a_{k}^{\prime}$ in another sequence, is replaced by the following constraint so that the executable time range of the event is equal in the both event sequences.

$$
T_{j_{\min }}=T_{k_{\min }}^{\prime} \wedge T_{j_{\max }}=T_{k_{\max }}^{\prime}
$$

Finally, timing constraints attached to each event is modified as follows.

For example, the timing constraints of events $e @ ? t 4$ and $b @ ? t 5$ of the following event sequence $P 12$ in Fig. 4

$$
c ? x 1 @ ? t 1[\ldots] ; ; @ ? t 4[t 4 \leq x 1] ; b @ ? t 5[t 5<t 1+t 4]
$$

are represented by the following expressions (4) and (5), respectively, with time variables $T_{c}, T_{e}$ and $T_{b}$ and integer variable $V_{x 1}$.

$$
\begin{array}{r} 
\\
T_{e}-T_{c} \leq V_{x 1} \\
\Rightarrow T_{e} \leq V_{x 1}+T_{c} \\
T_{b}-T_{e}<T_{c}+\left(T_{e}-T_{c}\right) \\
\Rightarrow T_{b}<2 T_{e}
\end{array}
$$

If we assume that the values of $T_{c}$ and $V_{x 1}$ vary between $\left[T_{c_{\min }}, T_{c_{\max }}\right]$ and $\left[V_{x 1_{\text {min }}}, V_{x 1_{\text {max }}}\right]$, respectively, the minimum value of the right-hand side of (4) is $V_{x 1_{\min }}+T_{c_{m i n}}$. Expression (4) shows that the upper bound of $T_{e}$ must be equal to or smaller than the value. Similarly, expression (1) shows that the lower bound of $T_{e}$ must be equal to or greater than $T_{c_{\text {max }}}$.

According to the above discussion and expression (4), the following constraint expression for event $e$ is finally derived.

$$
T_{c_{m a x}} \leq T_{e_{m i n}} \leq T_{e_{m a x}} \leq V_{x 1_{m i n}}+T_{c_{m i n}}
$$




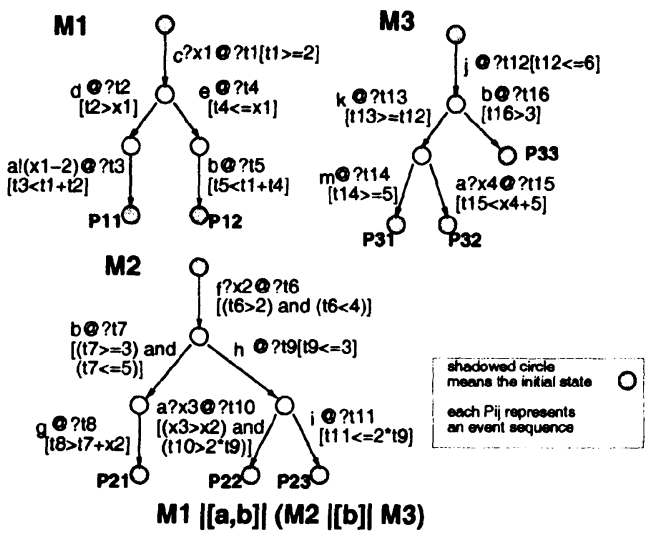

Figure 4 Example of concurrent periodic EFSMs

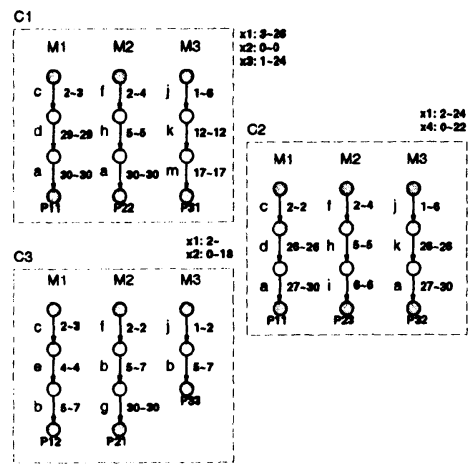

Figure 5 Executable time range of each event derived from the example in Fig.4

Similarly, for event $b$, the following constraint expression is derived from expressions (1) and (5).

$$
T_{e_{\max }} \leq T_{b_{\min }} \leq T_{b_{\max }} \leq 2 T_{e_{\min }}
$$

By solving a logical conjunction of the above constraint expressions for the following objective function (here, $w t_{i}, w v_{j}$ represent weighted coefficients),

$$
\max \sum w t_{i}\left(T_{i_{\text {max }}}-T_{i_{\text {min }}}\right)+w v_{j}\left(V_{j_{\max }}-V_{j_{\text {min }}}\right)
$$

we can obtain the executable time range of each event as well as the allowable value range of each integer variable. By setting appropriate values as weighted coefficients of the above objective function, we can derive larger executable time ranges for specified $\mathrm{I} / \mathrm{O}$ events and/or larger allowable value ranges for some variables.

Here, we applied the proposed technique to the example specification in Fig. 4. We used 30 units of time as the time interval of all EFSMs and used the following objective function for the ILP problem.

$$
\left(T_{a_{\max }}-T_{a_{\min }}\right)+\left(T_{b_{\max }}-T_{b_{\min }}\right)
$$

For three combinations $C 1, C 2$ and $C 3$ of synchronous event sequences among $M 1, M 2$ and $M 3$, the derived executable time ranges of all events are shown in Fig. 5. 


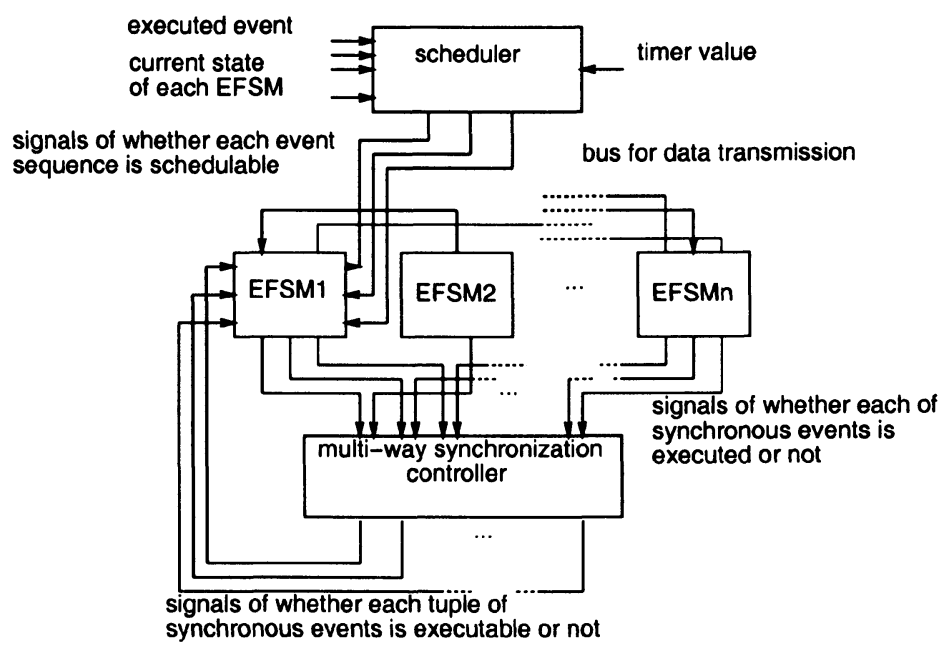

Figure 6 Architecture of the hardware circuit

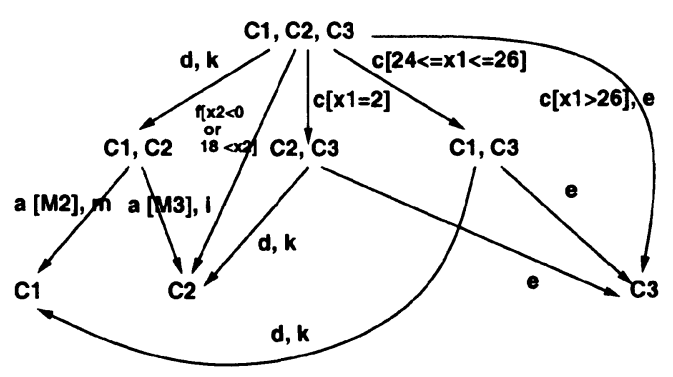

Figure 7 Scheduling tree of concurrent periodic EFSMs in Fig. 4

\section{HARDWARE CIRCUIT CONSTRUCTION 5.1. ARCHITECTURE}

As shown in Fig. 6, we compose the final circuit of the following three sub-modules: (1) sequential circuits corresponding to EFSMs working with the same clock, (2) a multi-way synchronization controller, and (3) a scheduler to indicate only schedulable event sequences to EFSMs at each time.

The sequential circuit implementing each EFSM has a state register for keeping the current state and a time register for keeping the time elapsed from the beginning of the current time interval (reset to zero in 
the initial state). The EFSM executes one of events outgoing from the current state at each clock cycle if it is executable. Otherwise, it stays in the same state.

The multi-way synchronization controller is constructed as (i) the executability checking part for checking whether each tuple of synchronous events is executable or not in each clock cycle, and (ii) the conflict avoidance part for selecting one of tuples which become executable but cannot be executed simultaneously. The above part (i) is constructed for each synchronous event tuple. Since each tuple becomes executable when all events in the tuple becomes executable, it is implemented as an AND circuit with $n$ inputs where each input connects to the signal from each EFSM in the tuple ( $n$ is the number of events in the tuple). For (ii), although there are several ways for selecting one of conflicting tuples, one of the simplest way is to put priority order among those tuples so that the tuple with the highest priority is selected. In that case, this part can be implemented as a priority encoder.

$[7,14]$ describe the details of the multi-way synchronization controller.

\subsection{SCHEDULER}

As we explained in Sect. 4, the executable time range of each event is derived for each event sequence or each combination of synchronous event sequences. So, the time range of an event included in several event sequences or in several combinations of synchronous event sequences is represented as a union of the derived time ranges. The executable time range of each event may change only when the following events are executed: (1) events on a branching state, and (2) synchronous events where each of them can be executed in different combinations of synchronous tuples. Thus, we can know the executable time range of each event by keeping the information about which branch is selected in each EFSM and on what tuple of synchronous event sequences is selected.

In the proposed technique, with the information about branches in each EFSM and the synchronous event tuples derived in Sect. 4.1, we construct the scheduling tree as shown in Fig. 7 where each node represents the current schedulable tuples of synchronous event sequences, and each edge represents an event execution which may reduce the number of schedulable tuples. The executable time range of each event and the allowable value range of each variable for each tuple of schedulable event sequences is derived as constant ranges (the lower and upper bounds) using techniques in Sect.4. So, using the ranges and the above scheduling tree, we construct the scheduler module as two parts: (1) a sequential circuit corresponding to a table which outputs the ranges 
of the executable time of each event and of the allowable value of each variable, depending on the current state in the scheduling tree (such a table can be easily implemented as ROM), (2) and a simple hardware component to check whether the current timer value and the input value are within those ranges.

\section{APPLICATION}

We have implemented a tool to derive the ranges for execution time of events and variables from a given specification described in E-LOTOS syntax. The tool uses the free-ware called lp_solve [9] to make a solution from the given ILP problems. We have also developed another tool to generate the RTL-level VHDL specification from the original specification and information derived with the first tool. With the tools and usual VHDL synthesis tools on the market, automated synthesis of hardware circuits from high-level specifications in the proposed concurrent periodic EFSMs model becomes possible.

As an example for evaluation, we have described a specification of a video play-back chip for video-conferences which can treat at most two video play-backs in a user terminal, and generated the corresponding hardware circuit with our tools.

In the example, we assume that (1) video streams from other two participants are transmitted to each chip (at each user) by packets (corresponding to video frames) where one packet is sent every 50 time units (each received packet is stored to the buffer in the chip), that (2) time interval between any two succeeding packets changes due to jitter on the network. Each received packet is decoded with an external module such as DSP and the decoded data is output to the display device, that (3) each user can dynamically join and leave the current session of the videoconference, and when a user leave the session, his/her video stream need not be processed in the chip, that (4) the external module can decode a video frame in 35 units of time, and that (5) the time period of all EFSMs is set to 100 units of time.

We modeled the system behavior as six concurrent periodic EFSMs consisting of (1) two displaying parts for drawing video frames in appropriate quality; (2) two admission parts for admitting the joining/leaving requests from two participants; (3) a decoding part for decoding a compressed video frame and outputting the decoded picture data (this operation is actually done by the external module like DSP); and (4) a control part for keeping the current number of participants.

In each EFSM for the displaying part, we have specified three branches where the first branch processes two video frames every time period 
(high-quality), the second one processes one frame in the same period (low-quality), and the final one does nothing in the case of user's leaving the session. One of the branches is dynamically selected depending on the number of users in the current session. When each EFSM obtains the compressed video frame data from the buffer, it transfers the data to the EFSM of the corresponding decoding part. Since there is only one EFSM for the decoding part and the decoding process takes 35 time units of time, when two displaying parts request decoding at the same time, one of them should be selected alternately (another one is delayed until the decoding of the first one is completed). In that case, the schedulable branch (with lower quality) should be dynamically selected in each EFSM using the proposed technique.

We have generated the corresponding RTL-level VHDL descriptions from the specification of the above example. The total time for the generation was about two seconds where most of the time is consumed by solving the ILP problem to obtain executable time ranges of events. We also synthesized the corresponding hardware circuit using Design Compiler of SYNOPSYS Corp. as the VHDL based synthesis tool on the market where we used the technology of CMOS $0.5 \mu \mathrm{m}$. As a result, the size of the synthesized circuit was about 3500 logic gates and its minimum clock period (representing the performance) was $150 \mathrm{nsec}$.

\section{CONCLUSION}

In this paper, we have proposed a concurrent periodic EFSMs model suitable to handle parallel and real-time processing, and a hardware implementation technique for the model. In the generated circuits, it is guaranteed that all of branches are schedulable in the initial state, and in any state at least one of the following branches is executable satisfying its timing constraints as long as the preceding $\mathrm{I} / \mathrm{O}$ events were executed in the time rages calculated in advance. We have also developed tools to generate the corresponding RTL-level VHDL descriptions from given specifications described in E-LOTOS syntax. The generated VHDL specifications can be treated in usual VHDL synthesis tools on the market to synthesize the hardware circuits. Through our experiment for a video playback chip generation, we have confirmed that the proposed model has sufficient expressiveness to describe typical real-time/multimedia systems and that the performance and size of the generated circuit is reasonable for practical use. In [7], we have shown network switches for ATM can be efficiently implemented as hardware circuits with the reasonable size and performance using our concurrent EFSM model where multi-way synchronization can be specified but timing constraints are 
not treated. The proposed method can also be used to synthesize such network switches requiring some timing guarantees.

Our future work includes integration of cost functions in the ILP problem to efficiently share limited resources among EFSMs.

\section{References}

[1] Csopaki, G. and Turner, K. J.: "Modeling Digital Logic in SDL", Proc. Joint Int. Conf. on 10th Formal Description Techniques and 17th Protocol Specification, Testing and Verification (FORTE/PSTV'97), pp. 367-382 (1997).

[2] Dave, B. P. , Lakshminarayana, G. and Jha, N. K. : "COSYN: Hardware-Software Cosynthesis of Embedded Systems", Proc. 34th Design Automation Conf. (DAC'g7), pp. 703-708 (1997).

[3] Fernandez, L. S. and Koch, G. and Madrid, N. M. and Vallejo, M. L. L. and Kloos, C. D. and Rosenstiel, W. : "Hardware-Software Prototyping from LOTOS", Design Automation for Embedded Systems 3, pp. 117-148 (1998).

[4] IEEE: "IEEE Standard VHDL Language Reference Manual", IEEE Std 1076-1993, (1994).

[5] ISO : "Information Processing System, Open Systems Interconnection, LOTOS - A Formal Description Technique Based on the Temporal Ordering of Observational Behaviour", ISO 8807 (1989).

[6] ISO : "Final Committee Draft 15437 on Enhancements to LOTOS", ISO/IEC JTC1/ SC21/ WG7, (1998).

[7] Katagiri, H., Yasumoto, K., Kitajima, A., Higashino, T. and Taniguchi, K.: "Hardware Implementation of Communication Protocols Modeled by Concurrent EFSMs with Multi-Way Synchronization", Proc. 37th Design Automation Conf. (DAC'2000), pp. 762-767 (2000).

[8] Kloos, C. D. and Moro, T. M. and Filho, G. R. and Lopez, A. M. : "VHDL Generation from a Timed Extension of the Formal Description Technique LOTOS within the FORMAT Project", Microprocessing and Microprogramming, 38, pp. 589-596 (1993).

[9] Michel, B., Jeroen, D. : “LP_SOLVE“, ftp://ftp.es.ele.tue.nl/pub/lp_solve.

[10] Sisto, R. : "A Method to Build Symbolic Representations of LOTOS Specifications", Proc. 15th IFIP Int. Symp. on Protocol Specification, Testing and Verification (PSTV$X V)$, pp. 331-346 (1995).

[11] Smith, J. and De Micheli, G.: "Automated Composition for Hardware Components", Proc. 35th Design Automation Conf. (DAC'98) (1998).

[12] Quemada, J., Larrabeiti, D. and Pavón, S. : "Compressing the State Space Representation of LOTOS Specifications", Proc. 6th IFIP Int. Conf. on Formal Description Techniques (FORTE'93), pp. 19-34 (1993).

[13] Wytrebowicz, J. : "Hardware Specification Generated from Estelle", Proc. 15th IFIP Int. Symp. on Protocol Specification, Testing and Verification (PSTV-XV), pp.435-450 (1996).

[14] Yasumoto, K. and Kitajima, A. and Higashino, T. and Taniguchi, K. : "Hardware Synthesis from Protocol Specifications in LOTOS", Proc. Joint Int. Conf. on 11th Formal Description Techniques and 18th Protocol Specification, Testing, and Verification (FORTE/PSTV'98), pp. 405-420 (1998). 